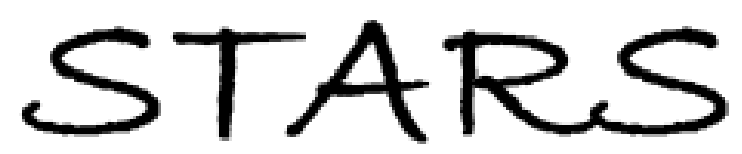

University of Central Florida

STARS

Retrospective Theses and Dissertations

1977

\title{
Quantitative Analysis of Thin Films by DC ARC Optical Emission Spectroscopy
}

Arnold W. Hogrefe

University of Central Florida

Part of the Chemistry Commons

Find similar works at: https://stars.library.ucf.edu/rtd

University of Central Florida Libraries http://library.ucf.edu

This Masters Thesis (Open Access) is brought to you for free and open access by STARS. It has been accepted for inclusion in Retrospective Theses and Dissertations by an authorized administrator of STARS. For more information, please contact STARS@ucf.edu.

\section{STARS Citation}

Hogrefe, Arnold W., "Quantitative Analysis of Thin Films by DC ARC Optical Emission Spectroscopy" (1977). Retrospective Theses and Dissertations. 342.

https://stars.library.ucf.edu/rtd/342

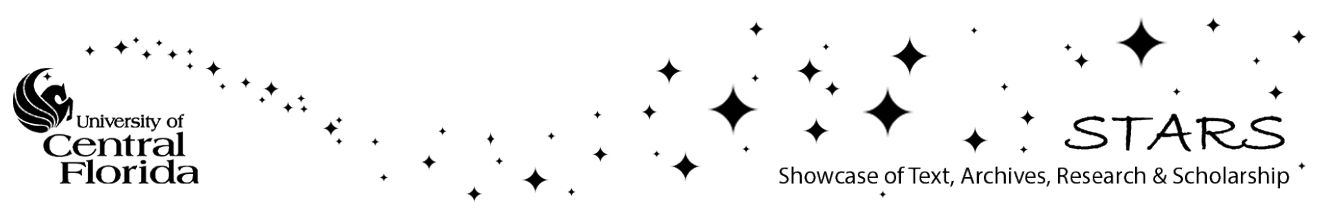




\section{QUANTITATIVE ANALYSIS OF THIN FILMS BY DC ARC OPTICAL EMISSION SPECTROSCOPY}

\section{BY}

ARNOLD W. HOGREFE

B.S., Bowling Green State University, 1970

\section{THESIS}

Submitted in partial fulfillment of the requirements for the degree of Master of Science: Industrial Chemistry in the Graduate Studies Program of the College of Natural Sciences of Florida Technological University

Orlando, Florida

1977 


\section{$\underline{\text { ABSTRACT }}$}

The use of DC arc optical emission spectroscopy (OES) for quantitative analysis of thin films deposited on graphite electrodes was investigated as a process control tool. Three binary systems were evaluated: nickel-chromium, phosphorous-silicon, and siliconaluminum. Sampling by direct deposition onto graphite electrodes placed in the deposition chamber with product runs proved to be a rapid, representative, and non-disruptive technique. Standard electrodes were prepared for each system either by evaporation of solutions of known concentration onto the tips of electrodes or by weighing out powdered standards of the appropriate concentrations. Standard curves were then prepared by burning multiple sets of standard electrodes in a DC arc of 15 amperes and obtaining intensity ratios of selected analytical line pairs.

Comparison of the OES technique with atomic absorption, electron microprobe, or gravimetric analysis of samples from the same deposition showed absolute agreement to within $\pm 3 \%$ for the nickel-chromium system, $\pm 0.3 \%$ for the phosphorous-silicon system, and $\pm 0.2 \%$ for the silicon-aluminum system. Maximum relative percent error for the techniques were $5 \%, 10 \%$ and $12.5 \%$ respectively. 


\section{ACKNOWLEDGEMENT}

The author wishes to gratefully acknowledge the management of Harris Semiconductor, a division of Harris Corporation, for their support and encouragement in the preparation of this report. 


\section{CONTENTS}

$\underline{\text { Page }}$

Introduction

Experimental $\quad 4$

Equipment $\quad 4$

Sample Film Preparation 4

$\begin{array}{ll}\text { Preparation of Standards } & 7\end{array}$

Excitation Conditions and Analytical Line Selection 9

$\begin{array}{ll}\text { Quantitation Procedure } & 10\end{array}$

Comparative Analysis Techniques 12

$\begin{array}{ll}\text { Results and Discussion } & 15\end{array}$

Nichrome 15

$\begin{array}{ll}\text { Phosphosilicate Glass } & 18\end{array}$

$\begin{array}{lr}\text { Silicon-Aluminum } & 22\end{array}$

Conclusion 31

References $\quad 32$ 


\section{LIST OF TABLES}

TABLE

TITLE

PAGE

I

Intensity Ratio Values for the Line Pair $3017.6 \mathrm{Cr} / 3003.6 \mathrm{Ni}$ Obtained from Standard Solutions

II

OES Analysis of $\mathrm{Ni}-\mathrm{Cr}$ Samples and Comparison to Atomic Absorption Spectroscopic Analysis

III

Intensity Ratio Values for the Line Pair 2535.6 P/2532.4 Si Obtained from Standard Solutions

IV

OES Analysis of P-Si Samples and Comparison to Electron Probe Microanalysis (EPMA)

Intensity Ratio Values for the Line Pair $2987.6 \mathrm{Si} / 3059.9 \mathrm{Al}$ Obtained from Powder Standards

VI

OES Analysis of Si-Al Samples and Comparison to Gravimetric Analysis 


\section{LIST OF FIGURES}

3 Chromium Calibration Curve Based on the $3017.6 \mathrm{Cr} / 3003.6 \mathrm{Ni}$ Line Pair

Correlation of OES and AAS Results for NickelChromium

Phosphorous Calibration Curve Based on the 2535.6 P/2532.4 Si Line Pair

Correlation of OES to EPMA Results and Calculated "Target" Values for Phosphorous-Silicon

Silicon Calibration Curve Based on the 2987.6 Si/3059.9 Al Line Pair

Correlation of OES and Gravimetric Results for Silicon-Aluminum 


\section{INTRODUCTION}

Quantitative analysis of vacuum deposited metal and chemical vapor deposited (CVD) glass thin films can present unique analytical problems as the "bulk" composition of films several microns to less than 100 angstroms thick must be measured. Obtaining representative samples in sufficient quantity for accurate analysis can in itself be a major obstacle, and sampling techniques must not disrupt the manufacturing process.

Thin films may be analyzed by suitable modification of classical wet methods and colorimetry, ${ }^{1-4}$ and by combination of wet and instrumental methods such as atomic absorption spectroscopy. ${ }^{3-6}$ However, these methods are time-consuming. They require dissolution of sample, often from multiple substrates, with high error potential in weighing. The determination itself can be complicated by interfering species or matrix effects. Electron beam methods are also useful for thin film analysis. ${ }^{7-12}$ However, for bulk thin-film composition such microanalysis can be difficult to quantitate due to lack of suitable standards. Small beam spots applied to nonhomogeneous films can lead to incorrect results, and computerized data treatment is often required to compensate for matrix effects and the statistics of the counting process. $\mathrm{x}$-Ray emission and fluorescence methods are perhaps the most useful quantitative analytical tool for thin films, and these methods are well documented. ${ }^{7-9,12-15}$

The purpose of this project was to develop a rapid, inexpen- 
sive, and suitably accurate analytical technique for the analysis of thin films. Due to excessive time requirements or lack of suitable instrumentation, all of the techniques mentioned previously were unacceptable for routine process control analysis. Since an emission spectrograph was available and the direct deposition of thin film samples on carbon electrodes was a potentially attractive sampling technique, the use of optical emission spectroscopy (OES) as a quantitative tool for analysis of thin films was chosen for investigation. Three thin films systems were evaluated: evaporated Nichrome, CVD phosphosilicate glass, and sputtered silicon-aluminum. Sample thin films were deposited on the tips of standard graphite electrodes appropriately situated in the vacuum evaporator, chemical vapor deposition chamber, or sputtering rig. In this manner, electrodes were conveniently included with thin-film production runs. They have proved entirely compatible with vacuum conditions utilized. ${ }^{16-17}$ Electrodes were then placed in the emission spectrograph for excitation. No micro-weighing or chemical pre-treatment of sample was necessary. Densitometry of the photographically recorded data, utilizing sensitive atomic lines, yielded film composition when compared to the OES data on properly prepared standard electrodes. Significantly, the mass of material deposited on electrodes was a little as $15 \mu \mathrm{g}$, considerably less than the amount of sample typically required for emission analysis. Although the composition of the "bulk" film must be determined, the techniques employed were necessarily in the realm of trace analysis. Accordingly, 
continuous DC arc excitation was the spectrographic method chosen, as it offers the greatest sensitivity for trace metals of any spectrographic source. ${ }^{18-19}$ OES has previously been applied to thin-film analysis by pre-concentration of dissolved samples or by peeling films from substrates. $20-21$ Dielman et al. 16 have used the technique for analysis of trace contaminants in aluminum thin films, but no attention has been given to quantitative analysis of thin films deposited directly on electrodes. 


\section{EXPERIMENTAL}

\section{Equipment}

An Applied Research Laboratories two-meter emission spectrograph, Rowland mount, with a 24,400 line/inch grating and $5.2 \AA / m m$ dispersion was used in this study. The optical range used was

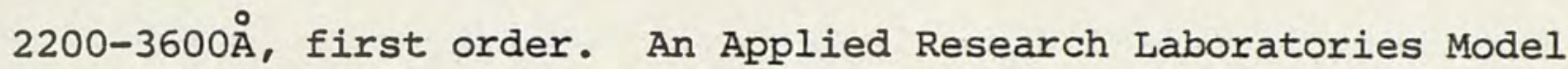
2255 scanning comparator densitometer was used for all film transmission readings.

All samples except silicon-aluminum standards were deposited on National L3960, 1/4 inch diameter flat end graphite electrodes and burned using National L3921, 1/4 inch diameter flat end counter electrodes and a continuous DC arc of 15 amperes. A $20 \mu$ slit and $3 \mathrm{~mm}$ analytical gap were used. Because of the massive electrodes and relatively short exposure times used, no adjustment of the analytical gap was necessary during arcing. No light reduction filters were employed.

Kodak Spectrum Analysis Film No. 1, D-19 developer and fixer were used with the manufacturer's recommended developing conditions. Sample Film Preparation

An electrode holder for obtaining nickel-chromium and siliconaluminum samples is shown in Figure 1. Graphite electrodes were clamped into a stainless steel collar so that only the electrode tip above the slot was exposed to the evaporation source or sputtering target. This assembly fit conveniently in one of the positions of 
the holder inside the deposition chamber. The thin films were deposited on the electrode tip with the same thickness and composition as the adjacent product. Thin film samples so obtained did not disrupt normal production procedures.

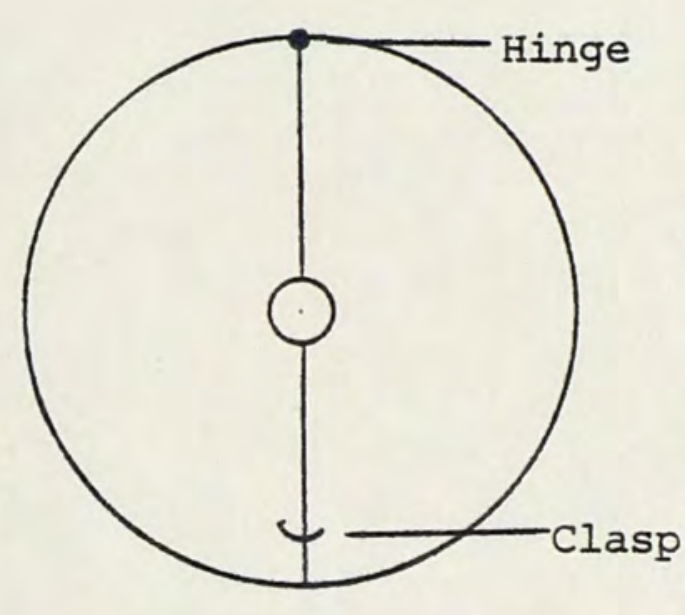

Top View

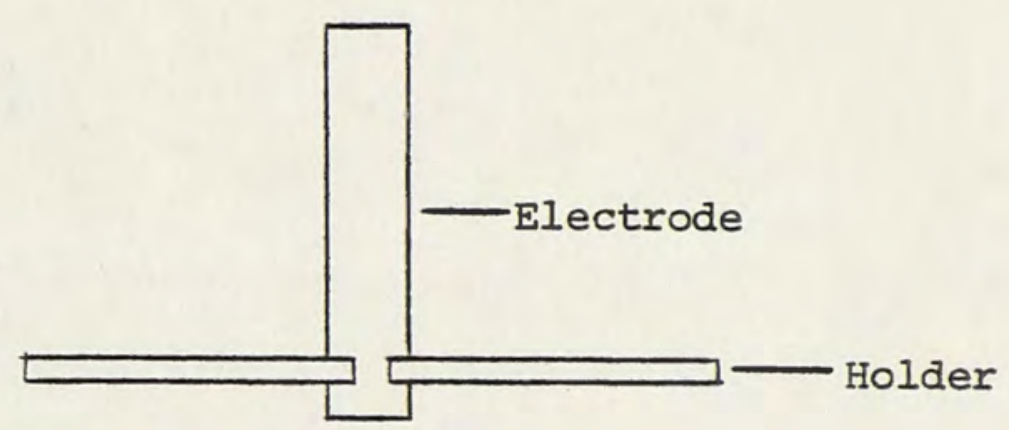

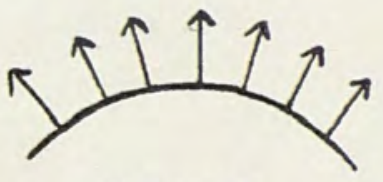

Source

Side View

\section{FIGURE 1}

NICHROME AND SILICON-ALUMINUM SAMPLING APPARATUS

PSG thin film samples were obtained by direct deposition of the glass onto the tip of a graphite electrode suitably placed in the CVD chamber with the product. The device used to hold the electrode in place is shown in Figure 2. A $1 / 4$ inch hole was drilled through the carbon support such that the base of the electrode rested on the graphite susceptor holding the product. This is important 
because the susceptor is heated and the amount of glass which deposits on a surface is directly related to the substrate temperature. The slight difference in temperature which may exist between the electrode tip and the actual product has a negligible effect on the analytical results. Typical samples were on the order of $250 \mu \mathrm{g}$ of PSG. Deviations from this mass did not adversely affect the results because the ratio of phosphorous to silicon, silicon being the internal standard, was used for the analysis.

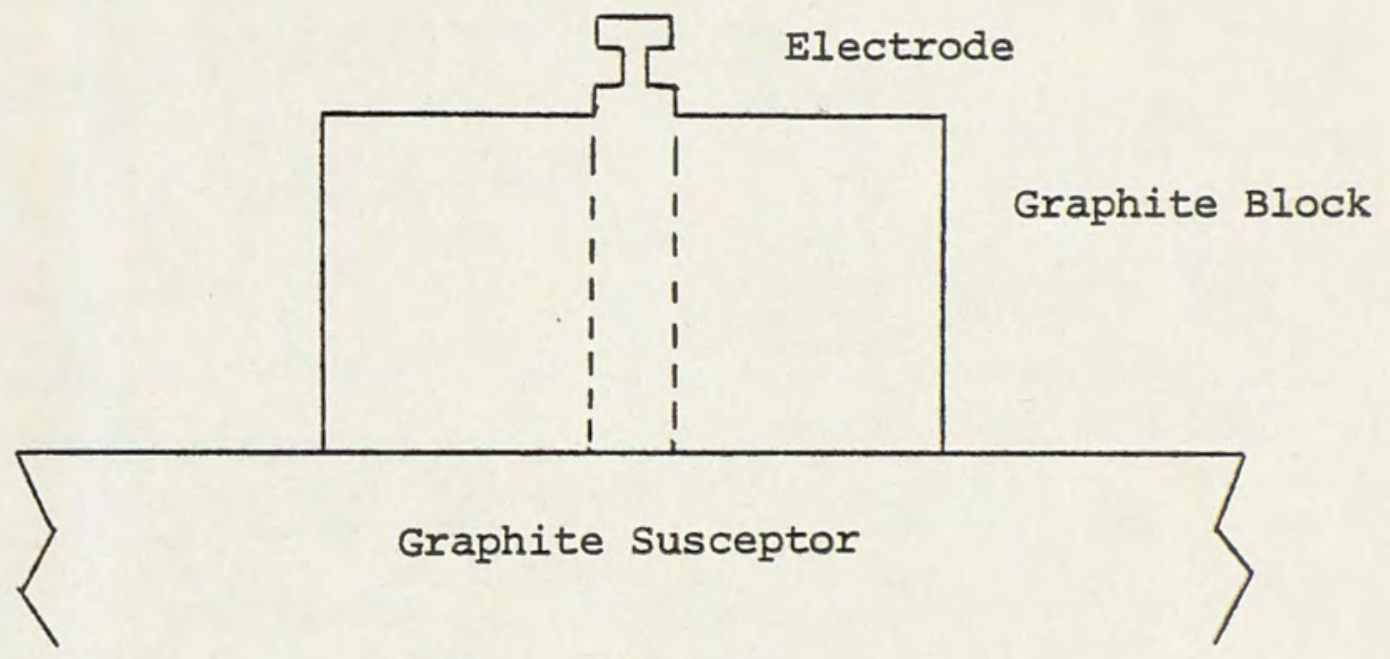

FIGURE 2

PSG SAMPLING APPARATUS 
Preparation of Standards

Nichrome. A stock solution of chromium was prepared by dissolving $\mathrm{K}_{2} \mathrm{Cr}_{2} \mathrm{O}_{7}$ (J. T. Baker reagent grade) in deionized water. A stock solution of nickel was prepared by dissolving the $99.999 \%$ metal (Alfa Inorganics) in warm nitric acid (J. T. Baker Electronic grade). Series of working standard solutions were prepared by appropriate dilution of aliquots of the stock solutions with deionized water. The final set of working standards was prepared by mixing of measured volumes of $\mathrm{Ni}$ and $\mathrm{Cr}$ standards to produce solutions $\mathrm{A}$ through G (Table I), such that $\mathrm{Cr}$ varied from $20 \%$ to $80 \%$ while Ni varied from $80 \%$ to $20 \%$, by weight. Each standard was prepared to contain a total mass of $\mathrm{Ni}$ and $\mathrm{Cr}$ equal to $15 \mu \mathrm{g} / \mathrm{ml}$.

Electrodes for calibration curves were prepared by evaporation of $1.00 \mathrm{ml}$ aliquots of standards $A-G$, applied dropwise via pipet to individual electrodes. Electrodes were held upright in a graphite block 1 inch thick and drilled to accept the 1/4 inch diameter electrodes. The block was placed on a hot plate maintained at $150^{\circ} \mathrm{C}$ during the dropwise addition of standard solution. Under these conditions, the solvent evaporated quickly without spattering and left a uniform deposit on the electrode tip. When complete, the standard electrodes contained a total mass of $\mathrm{Ni}$ and $\mathrm{Cr}$ approximately equal to the amount of metal on the sample electrodes. For each calibration run, triplicate electrodes were prepared for standards A-G.

Phosphosilicate Glass. Stock solutions of phosphorous and 
silicon were prepared by dissolving $\left(\mathrm{NH}_{4}\right)_{2} \mathrm{HPO}_{4}$ (J. T. Baker reagent grade) and sodium silicate (J. T. Baker reagent grade), respectively, in deionized water. A series of working standard solutions was prepared by diluting appropriate aliquots of the stock solutions with deionized water. Each $\mathrm{ml}$ of these standards, A-D (Table III) contained the approximate mass of $\mathrm{P}$ and $\mathrm{Si}$ present in the samples. For this procedure, $\mathrm{Si}$ served as the internal standard and was kept at a constant mass in all the standards. Since the samples were a phosphosilicate glass, the amounts of $\mathrm{P}$ and $\mathrm{Si}$ in the standards were adjusted to compensate for the oxygen in the samples. Calculations for the standards were based on the assumptions that the samples were composed of elemental phosphorous in $\mathrm{SiO}_{2} \cdot$ Although the samples are most likely $\mathrm{P}_{\mathrm{x}} \mathrm{O}_{\mathrm{y}}$ in $\mathrm{SiO}_{2}$, this simplification was justifiable since only weight percent phosphorous need be known for process control.

Evaporation of the standard solutions on the electrodes was performed in the same manner as for Nichrome. However, the hot plate temperature was lowered to $120^{\circ} \mathrm{C}$ to reduce spattering of the liquid. For each calibration run, triplicate electrodes were prepared from each standard.

Silicon-Aluminum. Use of solution standards proved unsuccessful for silicon-aluminum because of very low emission sensitivity for aluminum deposited from solution. This may have been caused by matrix effects introduced by the anions present or high volatility of the aluminum salt, resulting in loss of aluminum during the 
evaporation step.

Standards were, therefore, prepared from 6-9's silicon powder $(90-150 \mu)$ and 5-9's aluminum powder (90-150 $)$. Appropriate amounts of each powder were weighed in a plastic vial and mixed on a Wig-I-Bug mixer for thirty seconds. No mixing ball was included in the vial because it was found that the action of the ball embedded silicon in the walls of the plastic vial. Standards containing $1,2,3$, and 4 percent silicon by weight were prepared in this manner. For each standard, 0.25 to $0.35 \mathrm{mg}$ was weighed into a $1 / 8$ inch diameter, National L3975, shallow-cup graphite electrode in triplicate for each calibration run, and burned against a 1/8 inch diameter, National I4037 counter electrode. The same excitation conditions were used as for the 1/4 inch diameter electrodes. A comparison of results using $1 / 4$ inch versus $1 / 8$ inch diameter sample electrodes was made and will be discussed later in this report. Excitation Conditions and Analytical Line Selection

Optimum excitation conditions were determined by repetitive exposures of samples and standards at various settings. An exposure time of 20-30 seconds was found to be sufficient for total consumption of the sample, while consistently producing a background of 85-95 percent. Thus background corrections were found to be unnecessary for these techniques. 19

Nichrome. While there are abundant atomic lines of $\mathrm{Ni}$ and Cr, only the more sensitive arc lines are usable in this technique because of the small sample size. Excitations of standards prepared 
as above were carried out to identify lines for which intensities varied predictably as the known $\mathrm{Ni}$ and $\mathrm{Cr}$ concentrations. Of three possible Ni/Cr line pairs identified, the $3017.6 \mathrm{Cr} / 3003.6 \mathrm{Ni}$ pair was chosen based on the reproducibility of intensity values, calibration curve linearity, and their close proximity to each other thereby minimizing film-background effects during densitometry.

Phosphorous-Silicon. Since the sensitivity for phosphorous in the ultraviolet region is low and the number of lines are few, the analytical line choice was the most sensitive at $2535.6 \AA$. The close proximity of a suitable silicon internal standard line at 2532. $4 \AA$ made background differences negligible. Optimum excitation conditions for this line pair were determined by systematically exposing numerous sample and standard electrodes. An exposure time of thirty seconds was found to be optimum. All other excitation conditions were the same as those described previously.

Silicon-Aluminum. For the silicon-aluminum system, the line pair $2987.6 \mathrm{Si} / 3059.9 \mathrm{Al}$ was chosen by following the procedure described above.

\section{Quantitation Procedure}

Nichrome. For the two element Ni/Cr system, chromium is the analyte while nickel serves as the "internal standard". As has been described for spectrochemical calculations, ${ }^{22}$ the equations governing quantitation of this analysis are

$$
I_{\mathrm{Cr}}(3017.6)=\mathrm{F}_{\mathrm{Cr}}\left[\mathrm{C}_{\mathrm{Cr}}, \mathrm{C}_{\mathrm{Ni}}\right]
$$




$$
I_{\mathrm{Ni}}(3003.6)=\mathrm{F}_{\mathrm{Ni}}\left[\mathrm{C}_{\mathrm{Cr}}, \mathrm{C}_{\mathrm{Ni}}\right]
$$

where $I_{\mathrm{Cr}}$ and $I_{\mathrm{Ni}}$ are the integrated intensities for the indicated analytical line, $\mathrm{F}_{\mathrm{Cr}}$ and $\mathrm{F}_{\mathrm{Ni}}$ are functions derived from the spectral line intensities produced by the excitation parameters, and $\mathrm{C}_{\mathrm{Cr}}$ and $\mathrm{C}_{\mathrm{Ni}}$ are the concentrations of $\mathrm{Cr}$ and $\mathrm{Ni}$ atoms in the samples. For this two element system

$$
\mathrm{C}_{\mathrm{Cr}}+\mathrm{C}_{\mathrm{Ni}}=100 \text { percent }
$$

For continuous DC arc excitation, the absolute line intensities vary from one sample to the next because of instabilities inherent in the arc discharge. Thus, the absolute intensities can only be used when obtained by averaging a large number of runs. Repetitive samples can be run and the relative intensity values used to establish a working calibration curve. Using this approach, the intensity relationship becomes

$$
\frac{I_{C r}(3017.6)}{I_{N i}(3003.6)}=\frac{F_{C r}\left(C_{C r}, C_{N i}\right)}{F_{N i}\left(C_{C r}, C_{N i}\right)}
$$

$\mathrm{C}_{\mathrm{Cr}}$ and $\mathrm{C}_{\mathrm{Ni}}$ are not independent quantities, so Equation 3 holds and the expression becomes

$$
\frac{I_{\mathrm{Cr}}(3017.6)}{I_{\mathrm{Ni}}(3003.6)}=\frac{{ }_{\mathrm{Cr}}\left[\mathrm{C}_{\mathrm{Cr}}\left(100-\mathrm{C}_{\mathrm{Cr}}\right)_{\mathrm{Ni}}\right]}{{ }_{\mathrm{Ni}}\left[\mathrm{C}_{\mathrm{Cr}}\left(100-\mathrm{C}_{\mathrm{Cr}}\right)_{\mathrm{Ni}}\right]}
$$

where the quantity $\left(100-\mathrm{C}_{\mathrm{Cr}}\right)_{\mathrm{Ni}}$ represents the balance of film composition in addition to $\mathrm{Cr}$. Hence $\mathrm{C}_{\mathrm{Cr}}$ can be plotted as a function of 
the intensity ratio $I_{\mathrm{Cr}}(3017.6) / I_{\mathrm{Ni}}(3003.6)$ to yield a working standard curve for analysis of chromium content of thin films.

This-is a variation of the usual internal standard procedure in which one element remains at a relatively constant concentration and serves as a "standard" for analysis of the other.

Phosphosilicate Glass and Silicon-Aluminum. The phosphosilicate glass and silicon-aluminum procedures utilize the classical internal standard technique applied to very small samples. ${ }^{19,22}$ In the phosphosilicate glass technique, silicon serves as the internal standard for analysis of phosphorous, while in the silicon-aluminum procedure, aluminum serves as the internal standard for analysis of silicon. The major constituent was present at a concentration of greater than $90 \%$ over the range of the calibration curve for both these systems. Thus, it can be considered to remain essentially at a constant percentage relative to the variable unknown and can serve as the internal standard. The range of concentration of the standard curves was chosen to cover the range of anticipated thin film compositions.

Comparative Analysis Techniques

Nickel-Chromium. Atomic absorption spectroscopy (AAS) was employed to check the accuracy of the OES method. On selected depositions, $3 / 4$ inch $\times 1$ inch glass slides were placed adjacent to electrodes for the simultaneous collection of nichrome. Ten slides from each deposition were weighed, the deposited film dissolved in $0.01 \mathrm{M} \mathrm{CeSO}_{4}$ solution (prepared in $3 \mathrm{M}$ nitric acid), and the substrate 
slides dried and reweighed. Total sample weight was $0.8-1.0 \mathrm{mg}$. An analytical working curve for chromium was prepared from the standard stock solutions used for making the OES standards. The $\mathrm{CeSO}_{4}$ sample solutions were quantitatively diluted and the acidity of the standards adjusted to match that of the samples. AAS results for chromium in the selected samples were then obtained on a Jarrell-Ash Model JA 82-500 AA Spectrophotometer using a Cr hollow cathode lamp at a wavelength of $3578.7 \AA$. Percent nickel was not determined.

Phosphorous-Silicon. An electron microprobe method involving comparison of phosphorous X-Ray count data obtained from the thin film samples with count data from known standards was used to check the accuracy of the OES method. This was a modification of documented methods used previously. ${ }^{23-25}$ By taking phosphorous $\mathrm{X}$-Ray count data on standards of known thickness and composition, and then normalizing the counts to a standard thickness, a calibration curve was drawn. X-Ray counts and thickness measurements were then made on the unknowns, the count data normalized to the standard thickness, and percent phosphorous read from the calibration curve.

\section{Silicon-Aluminum. Gravimetric analysis for percent silicon} was the process control technique in use. Obviously, atomic absorption spectrophotometry would have been preferred, but the instrument available was not equipped for analysis of refractory elements. In the gravimetric procedure the thin film was collected on a suitable substrate, weighed, the aluminum selectively etched, and the substrate reweighed. The silicon was then mechanically swabbed off and the 
substrate again weighed. In this manner, the weight of silicon and aluminum in the films was determined. 


\section{RESULTS AND DISCUSSION}

Nichrome

Four triplicate analyses of standard electrodes done at different times gave the $I_{\mathrm{Cr}}(3017.6) / I_{\mathrm{Ni}}(3003.6)$ intensity ratio values for standards $A-G$ summarized in Table $I$. The increase in the standard deviation as percent $\mathrm{Cr}$ increases or decreases from $50 \%$ is due to increased microphotometer errors at high and low transmission readings. 26 At the extremes of the calibration curve, one element line is quite dark while the other is very light. The error in the analysis is, therefore, greatest at very high or very low chromium compositions, but is still acceptable for process control purposes.

Figure 3 is the calibration curve derived by plotting the average values for $I_{\mathrm{Cr}} / I_{\mathrm{Ni}}$ versus the respective $\mathrm{C}_{\mathrm{Cr}}$. This is the standard working curve for analysis of samples. The curve is valid as long as the roll of film in the spectrograph remains unchanged, and sample electrodes can be analyzed day after day until the "calibrated" film roll is consumed. Because of the possibility of slight changes in emulsions, a fresh roll of film is re-calibrated by analysis of standards B, D and F. Generally, however, the linearity or slope of the curve has not changed appreciably from one roll to the next. The shifts which have occurred would not have changed previous results by more than $\pm 0.5 \%$ chromium

Standard solutions prepared from $\mathrm{K}_{2} \mathrm{Cr}_{2} \mathrm{O}_{7}$ yielded more reproducible intensity ratio values than those prepared by dissolution of 
Intensity Ratio Values for the Line Pair $3017.6 \mathrm{Cr} / 3003.6 \mathrm{Ni}$ Obtained From Standard Solutions

Standard

Weight $: \mathrm{Cr}$

Run I

Run II

$\begin{array}{lll}0.34 & 0.46 & 0.60\end{array}$

0.86

0.95

0.78

0.92

0.78

0.96

1.2

1.14

0.48

0.52

0.87

0.93

0.80

1.03

1.24

0.78

0.95

1.16

1.23

0.64

0.57

0.78

1.02

1.24

0.99

1.19

0.88

1.26

0.79

0.58

$0.57 \quad 0.81$

0.96

1.22

1.59

Average

$$
0.35
$$

0.48

0.034

0.026

0.036

0.037

0.046

Average Dev.

Std. Dev.

$0.063 \quad 0.050$

$0.045 \quad 0.030$

0.043

0.045

0.061 


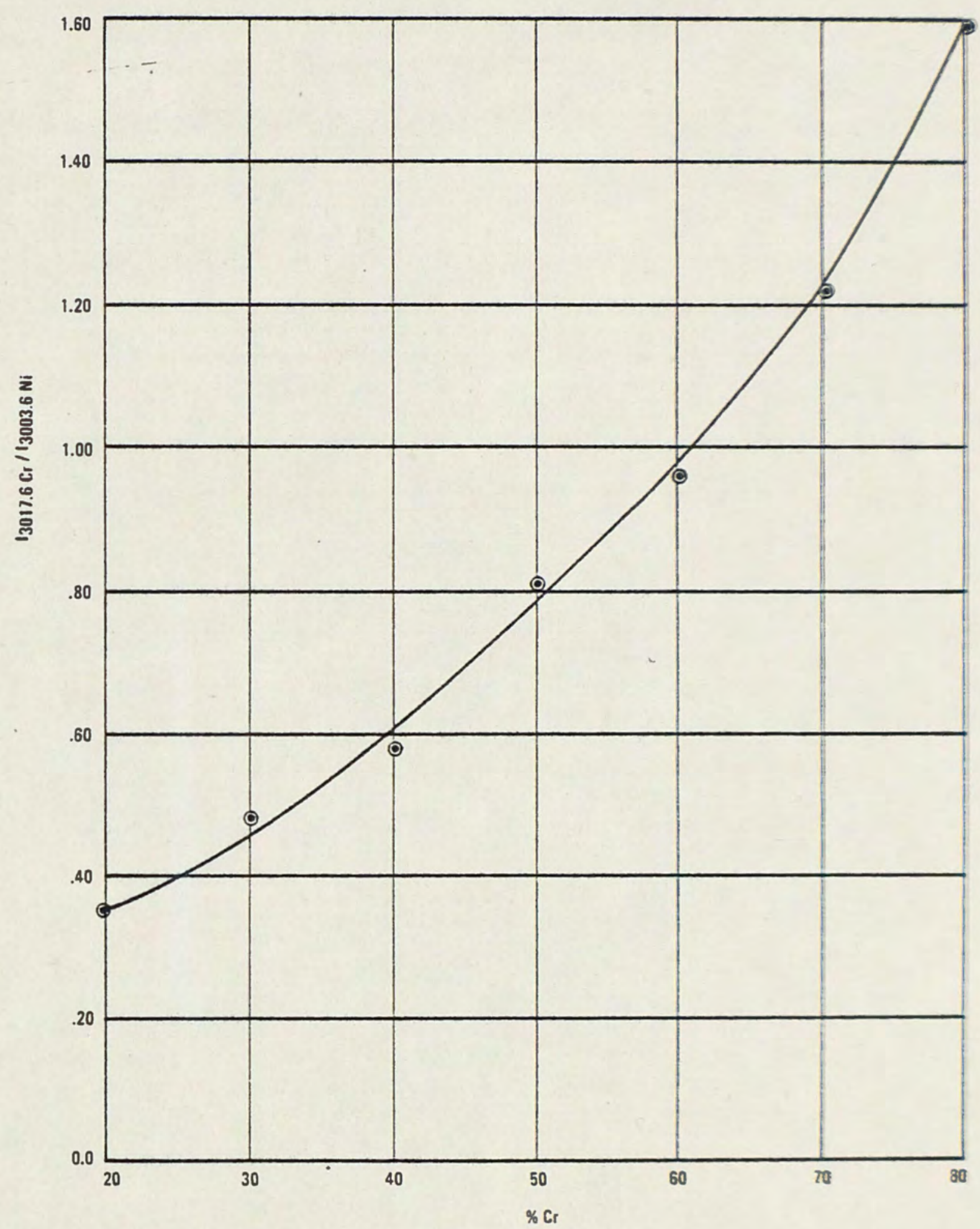

FIGURE 3

CHROMIUM CALIBRATION CURVE BASED ON

THE $3017.6 \mathrm{Cr} / 3003.6$ Ni LINE PAIR 
Cr metal. It is likely that the arc discharge was stabilized by the buffering action of potassium. 19,27 Although sample electrodes did not contain potassium, they exhibited arc stability comparable to the standards, possibly because of the uniformity of the film distribution over the electrode tip.

Quantitative analytical results for many nichrome film depositions of varying composition were completed as summarized in Table II. The percentage $C r$ values were obtained from the calibration curve in Figure 3. Reproducibility of results is evident from the data on multiple samples shown in Table II. Also listed are the AAS comparative analyses performed on many of the samples and the percent error of the OES results as compared to AAS.

An additional benefit of the OES method is its survey feature. After $\mathrm{Ni}$ and $\mathrm{Cr}$ line intensities were obtained, the film was scanned in the densitometer for presence of any other atomic lines which represent impurities. This provided a check on total thin film purity.

Figure 4 shows the correlation between OES and AAS results. Except for the data on the duplicate sample \#3, the relative error is less than $5 \%$ of the amount present. Duplication of the OES result suggests that the AAS determination for this particular sample may be in error.

Phosphosilicate Glass

Intensity ratios obtained from three sets of phosphoroussilicon standard electrodes are listed in Table III. A calibration 
TABLE II

OES Analysis of $\mathrm{Ni}-\mathrm{Cr}$ Samples and Comparison to Atomic Absorption Spectroscopic Analysis

\% $\mathrm{Cr}$ (OES)

34

37

$44,45^{\mathrm{a}}$

44

49

$57,57,57,57^{\mathrm{b}}$

60

65

71

74

77
\&Cr (AAS)

$\%$ error

$+20.3$

42

$+4.8$

49

0.0

68

$-4.4$

71

0.0

74

0.0

74

$+4.0$

a) Duplicate sample; 2 electrodes placed side-by-side in deposition chamber.

b) Quadruplicate sample; 4 electrodes placed side-by side in deposition chamber. 


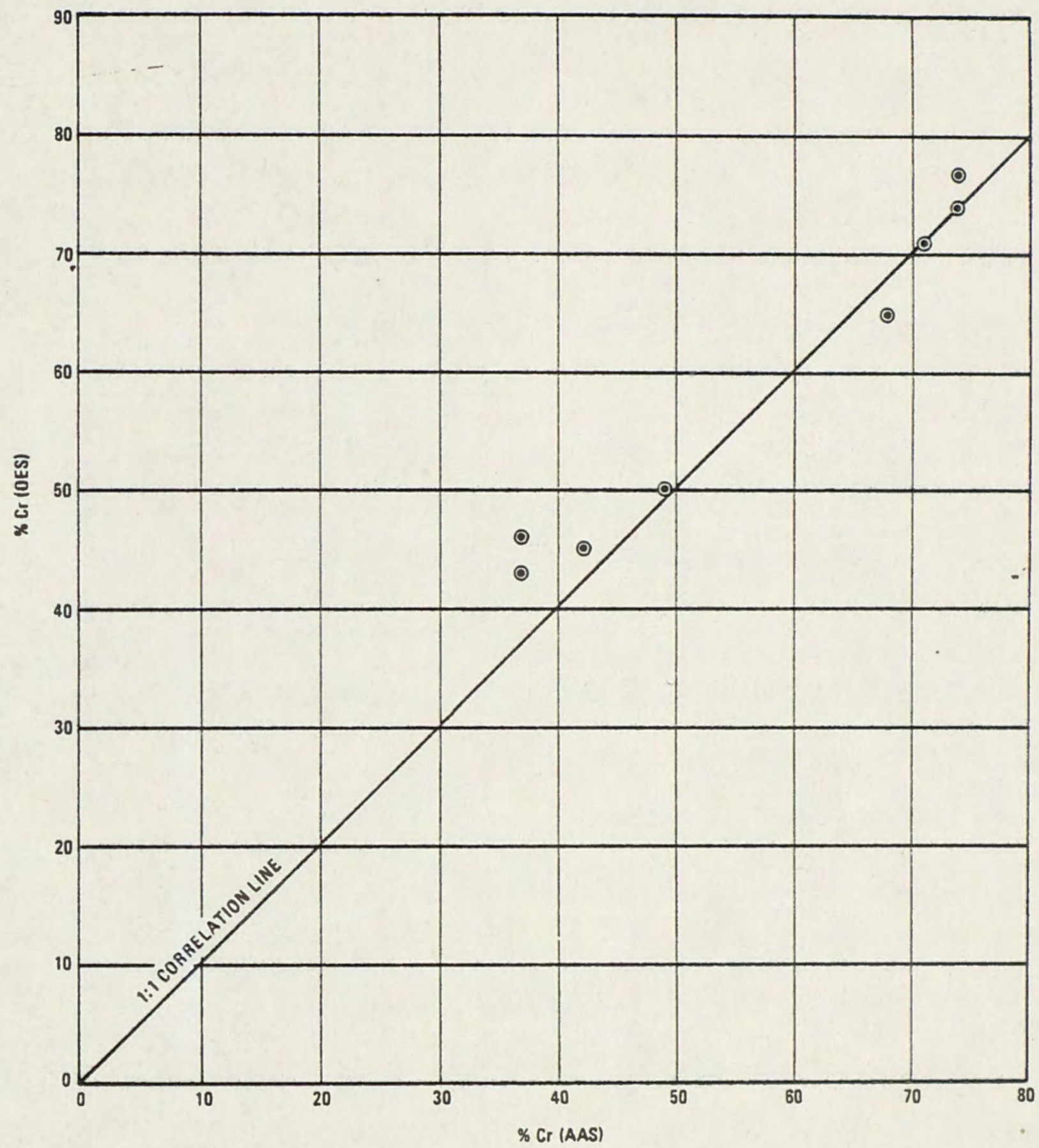

FIGURE 4

CORRELATION OF OES AND AAS RESULTS

FOR NICKEL - CHROMIUM 
Intensity Ratio Values For the Line Pair 2535.6 P/2532.4 Si Obtained From Standard Solutions

Standard

Weight $\% \mathrm{P}$

(Si remainder)

Run I

Run II

Run III

Average

Average

Deviation

Standard

Deviation

Least squares

data analysis
A

B

C

D
2.0
4.0
6.0
8.0

0.40

0.69

0.95

1.22

0.34

0.74

1.05

1.24

0.42

0.74

1.01

1.18

$\begin{array}{llll}0.29 & 0.75 & 1.04 & 1.23 \\ 0.30 & 0.69 & 1.09 & 1.39 \\ 0.32 & 0.57 & 1.07 & 1.20\end{array}$

\subsection{4}

0.73

0.92

1.36

0.37

0.78

0.93

1.15

0.37

0.76

0.96

1.17

0.36

0.71

1.00

1.24

0.05

0.06

0.06

0.08
0.04
0.05
0.06
0.06

0.39

0.68

0.97

1.27 
curve has been plotted from the averages of these data points in Figure 5. The line drawn represents the least squares fit of the data.

Comparisons of the OES technique to calculated doping levels, electron probe microanalysis (EPMA), and colorimetric analysis of side-by-side samples are shown in Table IV. The calculated values are based on flow rates of the reactants into the deposition chamber and are essentially the "target" concentration of the run. Colorimetric analysis using a modification of the phosphomolybdate method $^{1-4}$ was performed on only one sample because it proved to be excessively tedious and time-consuming. Figure 6 is a plot showing the correlation between OES analysis results and the EPMA results and target values. The largest error was $10 \%$ of the amount present, while typical relative errors were $4-8 \%$

\section{Silicon-Aluminum}

Intensity ratios obtained from four sets of silicon-aluminum standard electrodes are listed in Table V. A calibration curve has been plotted from the averages of these data points in Figure 7. The line drawn represents the least squares fit of the data. This is the working curve for analysis of thin film samples.

Comparisons of OES results to the sputtering target and gravimetric analysis are shown in Table VI. In the sputtering operation, the composition of the thin film produced closely resembles that of the sputtering target as long as proper procedures are followed. In several instances the target was not achieved, as seen from the OES 


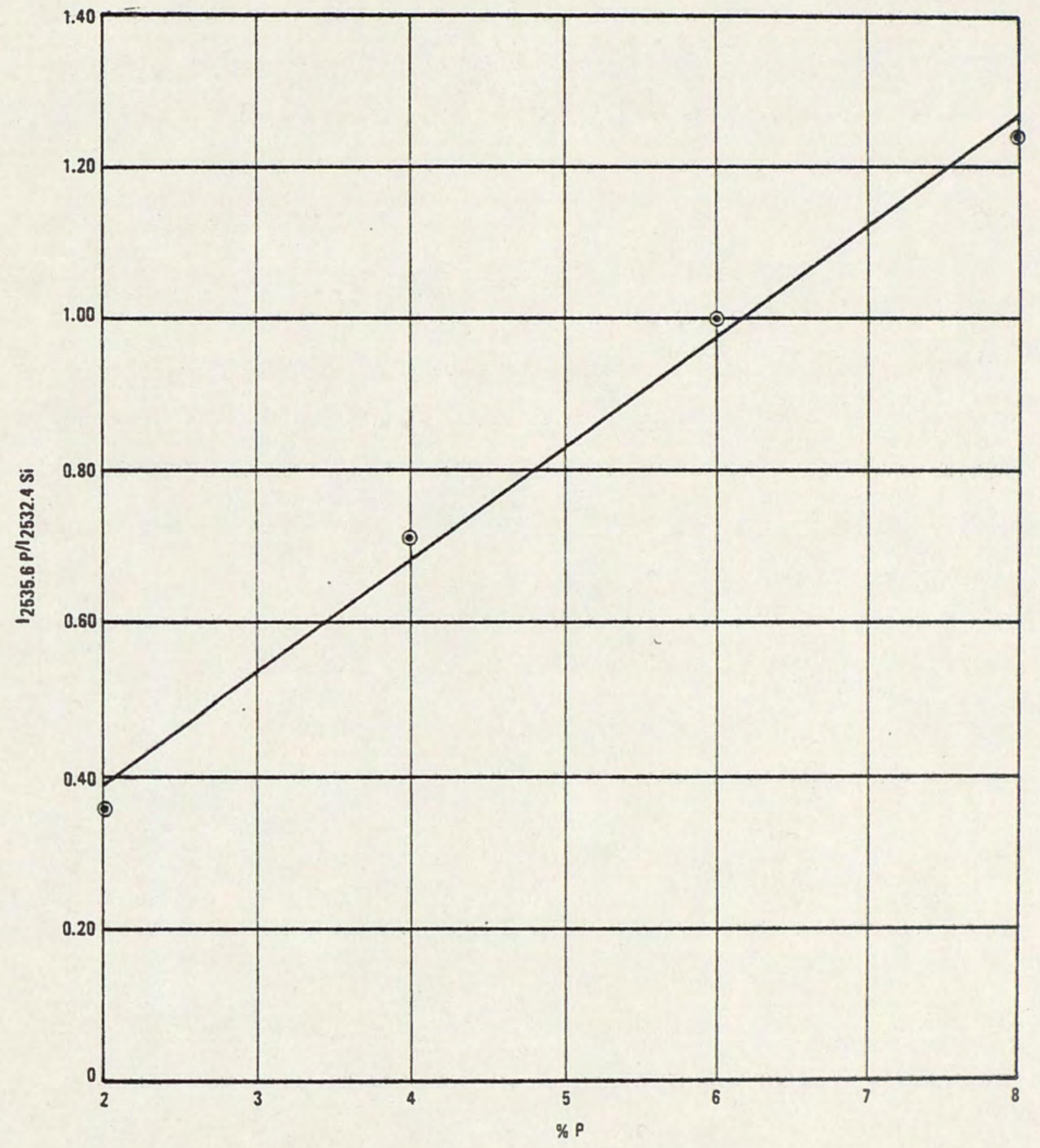

FIGURE 5

PHOSPHOROUS CALIBRATION CURVE BASED ON THE

2535.6 P / 2532.4 Si LINE PAIR 
TABLE IV

OES Analysis of P-Si Samples and Comparison to Electron Probe Microanalysis (EPMA)

\begin{tabular}{|c|c|c|c|c|}
\hline$\approx \mathrm{P} \quad(\mathrm{OES})$ & $\approx P \quad$ (EPMA) & $\begin{array}{l}\% \text { error } \\
\text { vs EPMA } \\
\end{array}$ & $\% \mathrm{P}$ (target) & $\begin{array}{l}\text { \% Deviation } \\
\text { from target }\end{array}$ \\
\hline $2.2,1.9^{a}$ & & & 2.0 & $+10.0,-5.0$ \\
\hline $5.1,5.5^{a}$ & & & 5.0 & $+2.0,+10.0$ \\
\hline $9 \cdot 6,9 \cdot 9^{a}$ & & & 10.0 & $-4.0,-1.0$ \\
\hline 6.1 & & & $6.0^{b}$ & +1.7 \\
\hline 5.3 & 5.7 & -7.0 & 5.5 & -3.6 \\
\hline 5.3 & 5.6 & -5.4 & 5.5 & -3.6 \\
\hline 5.5 & 5.2 & +5.8 & 5.5 & 0.0 \\
\hline 5.5 & 5.7 & -3.5 & 5.5 & 0.0 \\
\hline 6.3 & 5.8 & +8.6 & 6.0 & +5.0 \\
\hline 6.5 & 6.0 & +8.3 & 6.5 & 0.0 \\
\hline
\end{tabular}

a) Duplicate samples, two electrodes placed side-by-side in the deposition chamber.

b) Lengthy molybdate method on this sample gave $5.8 \%$ phosphorous. 


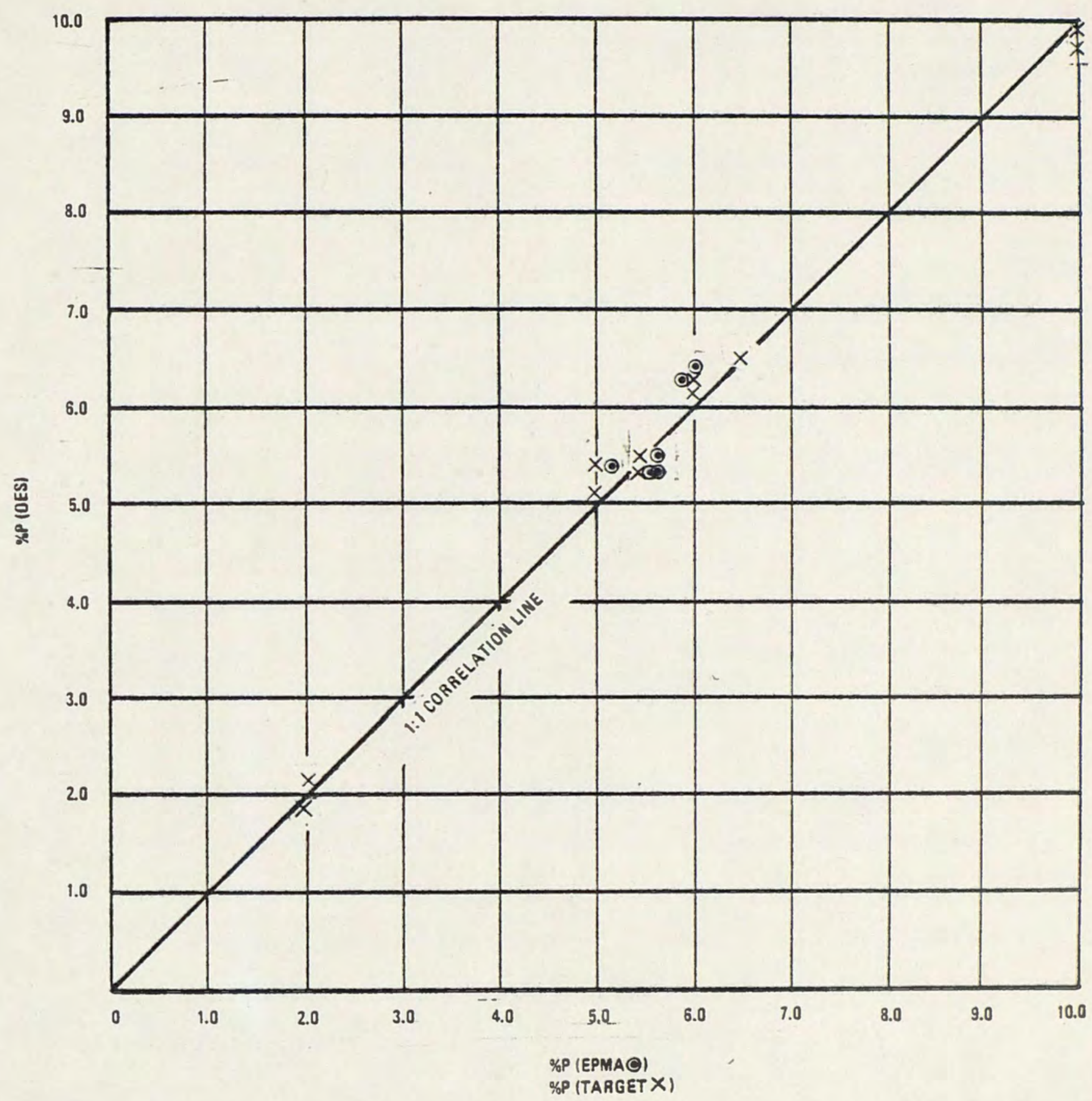


TABLE V

Intensity Ratio Values for the Line Pair $2987.6 \mathrm{Si} / 3059.9 \mathrm{Al}$ Obtained from Powder Standards

Standard

Weight $8 \mathrm{Si}$

(Remainder Al)

Run I

Run II

Run III

Run IV

Average

Av Deviation

Std Deviation

Least Squares

Data Analysis
A

B

C

D 


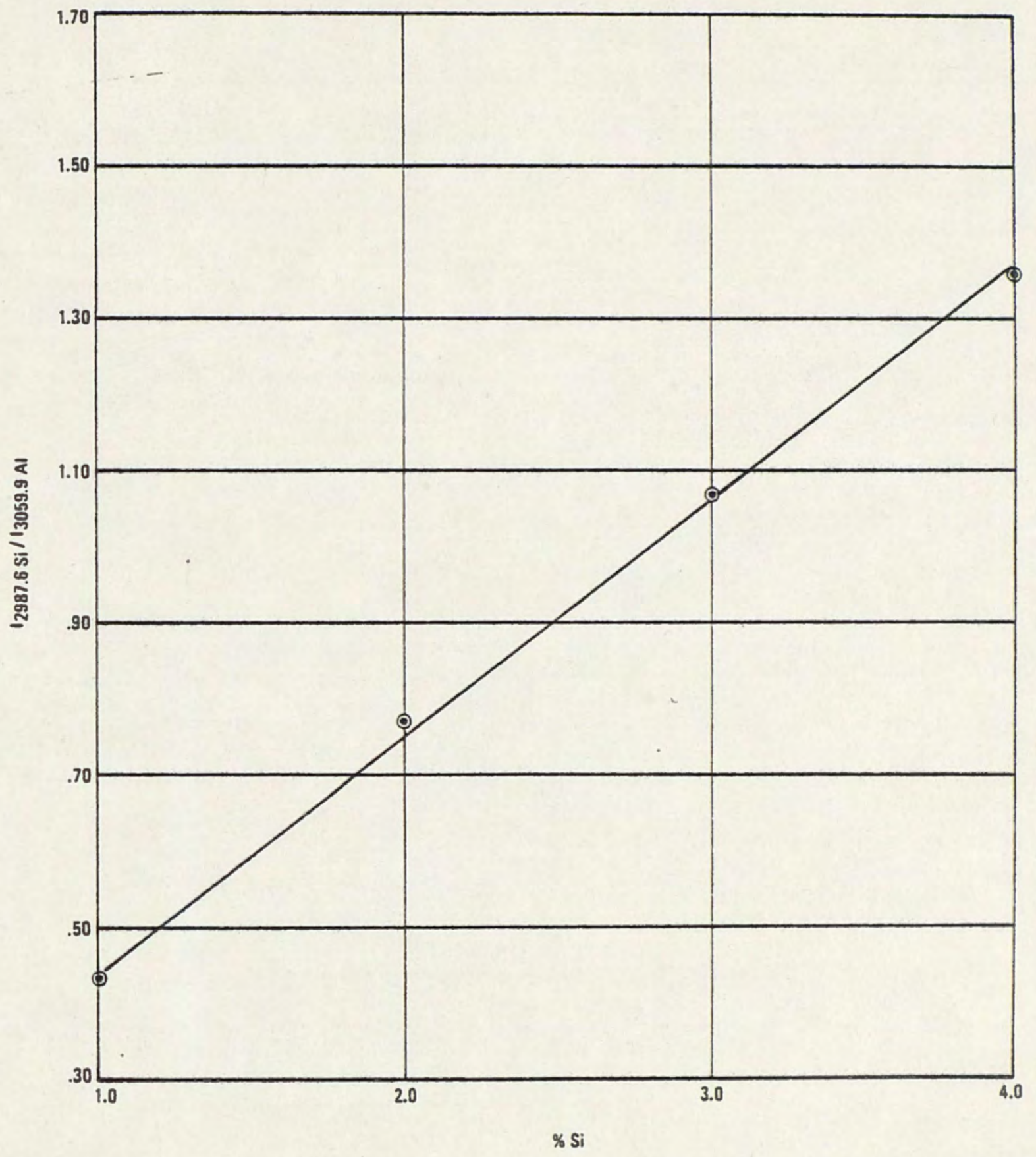

FIGURE 7

SILICON CALIBRATION CURVE BASED ON THE $2987.6 \mathrm{Si} / 3059.9$ AI LINE PAIR 
and gravimetric analysis results. It was later learned that these samples had been deposited by evaporation rather than sputtering and the exact process parameters to be used had not been established. Thus, these analyses proved useful to the process engineer for establishing the correct deposition conditions.

Because the Si-Al standards were burned in $1 / 8$ inch diameter cupped electrodes and the samples were collected on 1/4 inch diameter flat-end electrodes, the effect of electrode configuration on the results was evaluated. Samples were collected simultaneously on the 1/4 inch electrodes and on 1/2 inch diameter carbon disks. The sample on the disk was then scraped into a $1 / 8$ inch cupped electrode. The results for the two electrode types in Table VI show that there was no significant difference. This makes it possible to use disks as sample substrates in rigs which will not accommodate electrodes because of geometry restrictions.

Figure 8 is a plot showing the correlation between OES results using both electrode types and gravimetric results for percent silicon. The comparative analyses show absolute agreement to within $\pm 0.2 \%$ silicon. The maximum relative error was $12.5 \%$, with most analyses having a relative error of less than $8 \%$. 
TABLE VI

OES Analysis of Si-Al Samples and Comparison to Gravimetric Analysis

\& Si $\frac{\text { OES }}{1 / 4^{\prime \prime} \% \mathrm{Si}}\left(1 / 8^{\prime \prime}\right.$

electrode) electrode)

1.4

1.4

1.6

1.4

1.0

1.5

1.6

$$
1 \cdot 3,1 \cdot 4^{a}
$$

2.2

$$
\begin{gathered}
2.1 \\
1.6,1.8^{a} \\
0.9
\end{gathered}
$$

\% $\mathrm{Si}$

(Gravimetric)

1.6

1.5

1.0

$2.0^{b}$

$2.0^{b}$

2.0

$2.0^{\mathrm{b}}$

1.0

1.6

0.9
1.0

$\%$ error vs

\begin{tabular}{c}
$8 \mathrm{Si}$ \\
(Target) \\
\hline
\end{tabular}

gravimetric $1 / 4^{\prime \prime} 1 / 8^{\prime \prime}$

$-12.5 \quad-12.5$

$+6.7-6.7$

0.0
$-7.1,0.0$

$0.0 \quad-4.5$

$0.0,+12.5$

0.0

a) Duplicate disks placed side-by-side in the deposition chamber.

b) Target values not achieved due to deposition parameter errors. 


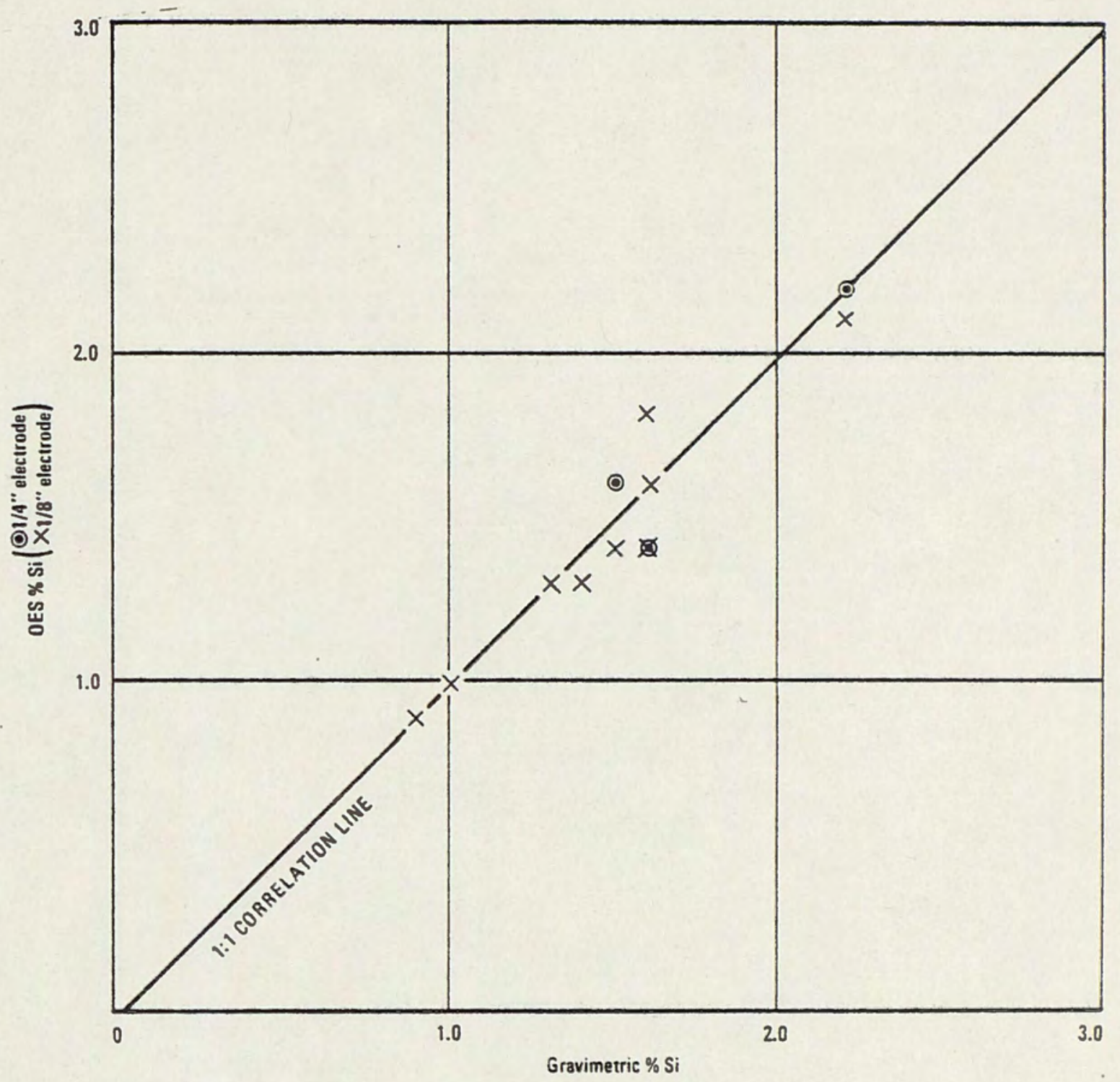

FIGURE 8

CORRELATION OF OES AND GRAVIMETRIC RESULTS

FOR SILICON - ALUMINUM 


\section{CONCLUSION}

The applicability of optical emission spectroscopy to analysis of thin films has been demonstrated for three binary systems. Many other similar binary systems would most probably be readily analyzed by modification of the techniques used. The direct deposition of sample thin films on graphite electrodes has reduced sample preparation and contamination problems, provided samples representative of product thin films, and has been non-disruptive of normal processing. These techniques have been proven acceptable for thin films deposited by thermal vacuum evaporation, ion sputtering, and chemical vapor deposition. Other thin film deposition methods, such as electroplating, could also be easily monitored by modification of these procedures.

The OES procedures described herein are well adapted to high volume, routine process control analysis. For the three systems evaluated, fifteen samples can be analyzed in only 90 minutes with accuracy sufficient for quality control purposes. 


\section{REFERENCES}

(1) Sandell, E. B., "Colorimetric Determination of Traces of Metals," Interscience, New York, N.Y., 1959, p 132.

(2) Furman, N. H., Ed., "Standard Methods of Chemical Analysis," D. Van Nostrand, Inc., Princeton, N.J., 1962, pp 703-713, 818-819, 960-963.

(3) Pratt, I. H., W. Weintraub and W. Wade, U.S. Army Electronics Command, Technical Report ECOM 2582, Fort Monmouth, N.J., April 1965.

(4) Pratt, I. H., W. Weintraub and W. Wade, U.S. Army Electronics Command, Technical Report 2419, Fort Monmouth, N.J., March 1964.

(5) Nohe, J. D., in "Physical Measurement and Analysis of Thin Films," Murt, E. M. and W. G. Guldner, Eds., Plenum Press, New York, N.Y., 1969, pp 138-147.

(6) Chandler, J. A. and M. S. Morton (1976), Anal Chem 48, 1316.

(7) Pliskin, W. A. and S. J. Zanin, in "Handbook of Thin Film Technology," Maissel, L. I. and R. Gland, Eds., McGraw-Hill, New York, N.Y., 1970, pp 11-1 to 11-54.

(8) Bishop, H. E. and D. M. Poole (1973), Appl. Phys. 6, 1143.

(9) Lutz, D., Naval Air Development Center, Report No. NADC-AE-6742, Johnsville, Warminster, Pa., Dec. 1967.

(10) Palmberg, P. W. (1972), J. Vac. Sci Technol. 9, 160.

(11) Evans, C. A., Jr. (1975), J. Vac. Sci. Technol. 12, (1), 268.

(12) Kern, W., T. D. Callinan Award Address, Electrochemical Society Meeting, Houston, Texas, May 1972.

(13) Spielberg, N. and G. Abowitz (1966), Anal. Chem. 38, 200.

(14) Zanin, S. J., J. C. Lloyd and G. E. Hooser, TR 22,186, IBM Corp., Components Division, Hopewell Junction, N.Y., 1965.

(15) Siggia, S., "Survey of Analytical Chemistry," McGraw-Hill, New York, N.Y., 1968, pp 23-25. 
(16) Dielman, J., A. W. Witmer, J. C. M. A. Ponsioeu and D. P. T. M. Damen (1973), Appl Spectrosc. 27, (5), 387.

(17) Beitel, G. A. (1971), J. Vac Sci Technol. 8, (5), 647.

(18) Morello, B., P. DiGregorio and G. Savastano (1974), J. Appl. Spect. $29,(1), 14$.

(19) Harvey, C. E., "Spectrochemical Procedures," Applied Research Laboratories, Glendale, Cal., 1950, pp 197-212, 238-239, 272297 , 340-342.

(20) Prokof'eva, F. T. (1968), Tr. Khim. Khim. Tekhnol. 2, 63.

(21) Shapilova, D. P. (1972), Vyschisl. Sist. 52, 180.

(22) ASTM Standard, Part 42, E-159 (1974).

(23) Pink, F. X. and V. Lyn (1968), Electrochem. Technol. 6, 258.

(24) Digiacomo, G. (1974), J. Electrochem. Soc. 121, 419.

(25) Esch, R. P., J. M. Eldridge, P. Balk and W. A. Pliskin, Abstract No. 95, Electrochemical Society Meeting, Detroit, Michigan, Fall, 1969.

(26) ASTM Standard, Part 42, E-305 (1974).

(27) Apel, C. T., Abstract No. 43, 27th Pittsburgh Conference, March $1-5,1976$. 